\title{
Entre-Parents: Initial Outcome Evaluation of a Preventive- Parenting Program for French-Speaking Parents
}

\author{
Sonia Lucia $\cdot$ Jean E. Dumas
}

Published online: 8 May 2013

(C) Springer Science+Business Media New York 2013

\begin{abstract}
Entre-Parents is the French adaptation of Parenting Our Children to Excellence, an eightsession group-parenting program for parents of preschoolers. An evaluation conducted in the Frenchspeaking part of Switzerland with 132 parents provides initial evidence for the community acceptability and efficacy of Entre-Parents. Program attendance was high (average of 6.6 out of the 8 sessions), and parents participated actively in sessions and expressed high levels of program satisfaction. Results indicate that, over time, the program contributed to more effective parenting practices, a reduction in parenting stress, an increase in family adaptability, and increases in children's social competence and reductions in their disruptive and anxious behaviors. Some of these benefits were stronger for parents who attended more sessions.
\end{abstract}

Keywords Prevention - Parenting - Parent training · Adaptation · Cultural differences · Group intervention

In all cultures, caring and skilled parents are essential to the development of socially and emotionally competent children (e.g., Desmet \& Pourtois, 2005; Rubin \& Chung, 2006). Not surprisingly, the family

S. Lucia · J. E. Dumas $(\bowtie)$

Department of Psychology, FPSE, University of Geneva, Bd du Pont d'Arve 40, 1211 Geneva 4, Switzerland e-mail: jean.dumas@unige.ch has long been the focus of programmatic efforts to stimulate children's healthy development and reduce their risk of behavioral or emotional problems in the early years. These efforts have led to the creation and refinement of parent training as a brief, cost-effective means of promoting positive parent-child interactions, regardless of whether the aim is to treat children who display behavioral or emotional problems (Geeraert, Van den Noortgate, Grietens, \& Onghena, 2004; Kazdin, 2005; Serketich \& Dumas, 1996), or to prevent such problems in children at risk (Dumas, Prinz, Smith, \& Laughlin, 1999; Piquero, Farrington, Welsh, Tremblay, \& Jennings, 2009; Prinz, 2012).

Although parent training has been implemented successfully in different contexts and countries, there are no research-based programs designed for French parents of preschoolers. The development of such programs is highly relevant, however, when one considers that large numbers of French-speaking parents are concerned about their children's ability to meet multiple challenges at home, at school, and beyond (Widmer, Kellerhals, Levy, Ernst, \& Hammer, 2003), and question their role and authority as they struggle to negotiate expectations and limits with their growing children (Sapin, Spini, \& Widmer, 2007).

This article describes the results of the first outcome study of Entre-Parents, a parenting program that aims to support parents of preschoolers through discussions and activities focused on practical childrearing issues. Entre-Parents is the French adaptation of Parenting Our Children to Excellence (PACE), a preventive 
intervention originally developed and tested by the senior author for English-speaking families (Dumas, Moreland, Gitter, Pearl, \& Nordstrom, 2008) and later adapted for Spanish-speaking families under the name of Criando a Nuestros Niños hacia el Éxito (CANNE) (Dumas, Arriaga, Begle, \& Longoria, 2010). Like its English and Spanish counterparts, Entre-Parents shares many of the theoretical assumptions of wellknown parenting programs, such as Positive Parenting Program (Triple-P; Sanders, 1999) and Parent-Child Interaction Therapy (PCIT; Zisser \& Eyberg, 2010), and promotes similar positive parenting practices. However, it is shorter and more modest in scope than Triple-P, which offers multiple intervention levels (from media campaign to intensive therapy) to reach targeted populations, groups or individuals. Also unlike PCIT, it is a group intervention that does not target individual families and does not involve live coaching of parent-child interactions.

Like PACE and CANNE, Entre-Parents is a manualized intervention led by trained and supervised group leaders and assistants. Groups consist of 10-15 parents who meet weekly for eight 2-h sessions. Each meeting gives parents repeated opportunities to address issues of concern to them and to support each other, as group leaders conduct guided discussions, use role plays, and show short video clips to cover a different topic in each session: (1) Guiding and encouraging our children, (2) Setting clear limits for our children, (3) Helping our children behave well at home and beyond, (4) Making sure our children get enough sleep, (5) Helping our children learn and grow, (6) Developing our children's self-esteem, (7) Helping our children do well at school, and (8) Parents, yes, but not only. Looking after ourselves.

Entre-Parents is the outcome of a systematic process of adaptation we have described elsewhere (Dumas \& Lucia, 2012). Briefly stated, adaptation proceeded in three steps. First, we consulted with community stakeholders (parents, service providers, and community leaders) to insure that the contents and format of each program session reflected major concerns of French-speaking parents and were sensitive to their cultural values and practices. Second, we adapted the English manual in light of this consultation process and translated it into French before evaluating the extent to which original and translated versions were similar (i.e., adaptation fidelity). Lastly, we translated measures not available in French and conducted a cross-language comparison of all measures to establish that they provide comparable data when administered in English and French.

Impetus for developing Entre-Parents came from repeated calls for a research-based intervention for French-speaking parents of preschoolers (Dumas \& Lucia, 2012) and was backed by evidence for the efficacy of the English and Spanish versions of the program. Studies show that parents attended both programs regularly, participated actively, and reported high satisfaction with the sessions' contents, format, and leadership. Most importantly, parents and children benefitted from the programs. This was indicated by increases from pre- to post-intervention and/or follow up on constructs such as parental efficacy and satisfaction and child social competence and social/ communication skills, as well as decreases on constructs such as parental harsh/inconsistent discipline, parenting stress, and child behavior problems. In both programs, these benefits were particularly evident for high attenders (i.e., parents who attended 4 or more of the 8 sessions), suggesting that "dosage" played an important role in the positive changes parents reported for themselves and their children (Begle \& Dumas, 2011; Dumas, Arriaga, Begle, \& Longoria, 2011).

In light of this prior research, we hypothesized that parents would: (1) attend Entre-Parents regularly, participate actively in sessions, and express high levels of satisfaction with the program; (2) report significant improvements in their parenting practices and stress, and in their children's adjustment at the end of the program and at a 3-month follow up; and (3) report benefits commensurate with the number of sessions they attended.

\section{Method}

Sample

Ten preschool/daycare centers were recruited during the 2010-2011 school year through the community stakeholders who had contributed to the program's adaptation. Located throughout the French-speaking region of Switzerland, all of these centers were part of the public school system. Once a center had agreed to host the program, a meeting was organized with the center director and staff to describe the program in 
detail, answer questions, and make practical arrangements to recruit families and host the sessions. EntreParents was advertised by placing posters and distributing flyers at the center and by announcing the program on a dedicated website. All advertisements stipulated that the only two requirements for parents to participate were to have one or more children between the ages of 3 and 6 , and to be the main persons responsible for their upbringing. Interested parents could either enroll online or complete and return a registration card to the center.

Of the 167 enrolled parents, 19 had scheduling conflicts or were no longer interested in the program when we offered them the opportunity to attend, 3 did not have a child in the 3-6 age range, and 15 were turned away from groups that were full (i.e., 17-18 parents registered even though we had asked each center to cap registrations at 15). This left a sample of 130 parents, of whom 30 were couples (96 mothers, 32 fathers, and 2 grandmothers). As we interviewed one parent per couple only, 100 parents completed the Time 1 (T1) interview. The same number completed the Time 2 (T2) interview and 98 completed the Time 3 (T3) interview 3 months later (yielding a $98 \%$ retention overall). Table 1 summarizes the sample's sociodemographic characteristics. The 100 children targeted by the program included 34 girls and 66 boys, with a median age of $4(M=4.06, S D=.95$, range 3-6). The sample included a significantly larger proportion of boys than girls (Binomial test, $p<.01)$. The 100 parents included 89 mothers, 9 fathers, and 2 grandmothers with a median age of 36 ( $M=36, S D=5.73$, range $24-68$ years). The majority were married, lived with the child's other parent, or lived in blended families. Although most of the children were born in Switzerland, a majority of their parents had immigrated relatively recently. Parents were generally working outside the home, even though most of them had a part-time job. Official statistics suggest that the sample's income level was comparable to that of other Swiss families but that its education level was high, considering that about $24 \%$ of Swiss residents have a university degree. ${ }^{1}$

\footnotetext{
${ }^{1}$ http://www.bfs.admin.ch/bfs/portal/fr/index/themen/01/04/ blank/01/03/01.html, http://www.bfs.admin.ch/bfs/portal/fr/ index/themen/15/01/key/blank/01.html.
}

Table 1 Sample's sociodemographic characteristics

\begin{tabular}{|c|c|c|}
\hline \multirow[t]{2}{*}{$\begin{array}{l}\text { Family } \\
\text { composition }\end{array}$} & $\begin{array}{l}\text { Two-parent (includes married, } \\
\text { cohabiting and blended } \\
\text { families) }\end{array}$ & $88.9 \%$ \\
\hline & One-parent & $11.1 \%$ \\
\hline \multirow[t]{2}{*}{ Parents' origin } & Born in Switzerland & $60.0 \%$ \\
\hline & Born outside Switzerland & $40.0 \%$ \\
\hline \multirow{3}{*}{$\begin{array}{l}\text { Parents' migration } \\
\text { background }^{\mathrm{a}}\end{array}$} & Swiss & $37.0 \%$ \\
\hline & 2nd generation & $25.0 \%$ \\
\hline & 1st generation & $38.0 \%$ \\
\hline \multirow{5}{*}{$\begin{array}{l}\text { Parents' } \\
\text { employment } \\
\text { status }\end{array}$} & Employed (full- or part-time) & $77.0 \%$ \\
\hline & Homemakers & $18.0 \%$ \\
\hline & Unemployed & $2.0 \%$ \\
\hline & Disabled & $1.0 \%$ \\
\hline & Student & $2.0 \%$ \\
\hline \multirow[t]{3}{*}{ Parents' education } & $\begin{array}{l}\text { Compulsory education (up to } \\
\text { age 16) }\end{array}$ & $6.0 \%$ \\
\hline & Upper secondary education & $48.0 \%$ \\
\hline & Post-secondary education & $46.0 \%$ \\
\hline \multirow[t]{4}{*}{ Family income } & $<\mathrm{CHF}^{\mathrm{b}} 72,000 /$ year & $14.3 \%$ \\
\hline & CHF 72,000-143,999/year & $62.2 \%$ \\
\hline & CHF 144,000-215,999/year & $17.3 \%$ \\
\hline & $>$ CHF 216,000/year & $6.1 \%$ \\
\hline
\end{tabular}

These characteristics are for a sample of 100 participants

a A parent was considered Swiss if born in Switzerland or abroad of two parents who were born in Switzerland. A parent was considered a second generation migrant if born in Switzerland of one or both parents born abroad. A parent was considered a first generation migrant if born abroad of one or both parents born abroad

${ }^{\mathrm{b}} \mathrm{CHF}=$ Swiss francs

\section{Procedures}

All procedures were approved by the Institutional Review Board of the University of Geneva's Faculté de Psychologie et des Sciences de l'Education.

\section{Staff Training}

Prior to the start of the study, we recruited group leaders, group assistants, and interviewers who spoke French fluently. Group leaders and assistants $(n=12$, some of whom served as leader for one group and assistant for another) all had professional experience in psychology, education or healthcare. They participated in a three-day training workshop that focused on mastering and maintaining fidelity to the program's process and content. Content training pertained to the 
topics to be covered in each session, and to their rationale, presentation, and supporting materials (e.g., CDs, posters, handouts). It also emphasized the importance of maintaining the schedule in order to cover all session activities in the allotted time. Process training stressed the necessity of involving parents in all aspects of each session and focused on group management and on effective communication skills. It provided specific instruction on how to present the program clearly, manage transitions and interruptions, deal with resistance and opposition, listen attentively, show concern and respect for different opinions, avoid criticism and unsolicited advice giving, and provide frequent positive feedback (Dumas, Lynch, Laughlin, Smith, \& Prinz, 2001). Training was both didactic and "hands-on," as it required all trainees to conduct group sessions using workshop participants as parents. Following training, group leaders were supervised regularly and recorded during each session they conducted to ensure that they delivered the program as designed (see Fidelity below). Interviewers underwent individual training to ensure that they could establish rapport with parents, field questions without straying from the wording of the interview, and work through the interview in a timely manner. They were also supervised regularly following training.

\section{Parenting Groups}

Group leaders were responsible for delivering the entire program to the same group of parents, with the help of assistants who provided programmatic and logistical support (e.g., talking to parents individually before/after sessions, preparing food, setting up videos, taking part in role plays). Attendance at the 10 Entre-Parents groups that were offered was free. All groups met in the evening. At each session, free on-site childcare was provided and parents received a free snack while their children received a free meal.

\section{Interviews}

Interviewers contacted parents by phone to schedule all interviews, which usually took place at the preschool/daycare center or at the parent's home. In two-parent households, the same parent completed all three interviews. Interviewers followed a script to conduct each interview, which lasted 45-60 min at T1 and 35-45 min at T2 and T3. When parents had more than one child in the targeted age range $(n=24)$, parents were instructed to select one of them and to report on that child for all three interviews. Parents received a grocery store or bookstore gift card worth 30 Swiss francs for each interview.

\section{Measures}

To test the three hypotheses describe above we collected measures of program attendance, participation, and satisfaction, and of child and parent/family functioning. Measures were obtained from group leaders and assistants at the end of each session, and from parents at three time points: Time 1 (before the program), Time 2 (at the end of the program), and Time 3 (at follow-up). They included: (1) measures of program attendance, participation in sessions, and parental satisfaction, (2) sociodemographic measures, (3) measures of parenting practices, parenting stress, and family functioning, and (4) measures of child coping competence, and behavioral and emotional problems. All activities-data collection and program-were conducted in French.

Group leaders and assistants completed measures of attendance and participation at the end of each session. Parents completed a measure of program satisfaction at the end of sessions 4 and 8 (or after those sessions for parents who were absent). Parents provided sociodemographic information at $\mathrm{T} 1$ and completed measures of parent/family functioning and of child adjustment at all three periods. These measures were all commonly used, validated measures that we translated into French as necessary and pilot tested for use in this study (Dumas \& Lucia, 2012).

\section{Attendance and Quality of Participation}

After each session, the group leader and assistant recorded attendance and independently rated each parent's quality of participation on a 7-item scale ranging from 1 to 5 (with specific definitions for each anchor point). The 7 items are described in Table 2. Each anchor point had clear definitions that leaders and assistants had been trained to use. As their ratings were internally consistent (average $\alpha=.88$ for leaders and .87 for assistants) and significantly correlated (with $r$ 's ranging from .34 to .63 across sessions, all $p<.001$ ), they were averaged to yield a single quality of participation score per parent. 
Table 2 Mean ratings and standard deviations $(S D)$ of parental satisfaction with the program

\begin{tabular}{lll}
\hline & Mean & $S D$ \\
\hline $\begin{array}{l}\text { Group leader and assistant } \\
\text { The group leaders respect my }\end{array}$ & 4.80 & .40 \\
$\quad$ values and opinions & & \\
$\quad \begin{array}{l}\text { The group leaders communicate clearly } \\
\quad \text { in ways I can understand }\end{array}$ & 4.77 & .45 \\
$\quad \begin{array}{l}\text { The group leaders are motivated } \\
\text { and enthusiastic }\end{array}$ & 4.83 & .37 \\
$\begin{array}{l}\text { Program relevance and benefits } \\
\text { The topics covered in the program } \\
\text { are important }\end{array}$ & 4.64 & .51 \\
$\quad \begin{array}{l}\text { The topics covered in the program } \\
\text { are useful to me and my family }\end{array}$ & 4.42 & .60 \\
$\begin{array}{l}\text { I put the program's ideas/suggestions } \\
\text { in practice }\end{array}$ & 4.27 & .58 \\
$\begin{array}{l}\text { Program recommendation } \\
\text { I would recommend this program } \\
\text { to other parents like me. }\end{array}$ & & \\
\hline
\end{tabular}

\section{Program Satisfaction}

Parents completed the French version of the PACE Social Validity Survey (unpublished) twice, at the midpoint and end of the program. This 14-item survey, which was internally consistent $(\alpha=.78)$, measured the extent to which parents were satisfied with their group leader, found the program relevant and beneficial, and would recommend the program to other parents (e.g., "The group leaders talk with me in ways I can understand." "The goals of the program are important to me."). Ratings were averaged across parents to assess each of these dimensions, with higher numbers indicating higher levels of satisfaction.

\section{Parental/Family Functioning}

Parents completed measures of parenting practices, parenting stress, and family functioning at T1, T2, and T3. The Parenting Practices Interview (PPI; WebsterStratton, 1998) is an adaptation of the Oregon Social Learning Center's Discipline Questionnaire for use with parents of preschoolers. A 37-item abbreviated version assesses positive/appropriate discipline (average $\alpha=.64$, range .61-.66; e.g., "How often do you praise or reward your child when your child plays nicely alone or with another child?") and harsh/ inconsistent discipline (average $\alpha=.73$, range .70.78; e.g. "How often does the kind of punishment you give your child depend on your mood?"). The Parenting Stress Index-Short Form (PSI-36; Abidin, $1997)$ is a 36-item measure that yielded three indices of parenting stress labeled 'parental distress' (average $\alpha=.85$, range .83-.87; e.g., "I feel alone and without friends."), 'parent-child dysfunctional interaction' (average $\alpha=.80$, range .74-.85; e.g., "My child rarely does things for me that make me feel good."), and 'difficult child' (average $\alpha=.88$, range .87-.89; e.g., "Sometimes my child does things that bother me just to be mean."). The Family Adaptability and Cohesion Evaluation Scales (FACES-III; Olson, Portner, \& Lavee, 1985) consist of 20 items that provided ratings of family functioning on the broad dimensions of 'adaptability' (average $\alpha=.70$, range .67-.74; e.g., "We shift household responsibilities from person to person.") and 'cohesion' (average $\alpha=.76$, range .70.80; e.g., "When our family gets together for activities, everybody is present.").

\section{Child Adjustment}

Parents also completed measures of their children's adjustment at each of the three time points. The Social Competence and Behavior Evaluation Scale-Short Form (SCBE-30; LaFreniere \& Dumas, 1996) is a 30 -item instrument that we used to obtain ratings of child social competence (average $\alpha=.69$, range .65.78 ; e.g., "Comforts or assists another child in difficulty."), anger-aggression (average $\alpha=.83$, range .81-.86; e.g., "Irritable, gets mad easily."), and anxiety-withdrawal (average $\alpha=.73$, range .69.78; e.g., "Inactive, watches others."). The Eyberg Child Behavior Inventory (ECBI; Boggs, Eyberg, \& Reynolds, 1990) is a 36-item instrument that assesses child disruptiveness along two dimensions: an intensity dimension that evaluates the extent of disruptive conduct (average $\alpha=.89$, range .87-.92) and a problem dimension that specifies the number of conduct problems the child presents (average $\alpha=.90$, range .88-.92). Sample items include "Refuses to obey until threatened with punishment" and "Physically fights with sisters and brothers." Finally, a 15-item measure consisting of three of the five subscales of the Strengths and Difficulties Questionnaire (SDQ; Goodman, 1997) was administered to obtain ratings of child prosocial behavior (average 
$\alpha=.68$, range .66-.71; e.g., "Often offers to help others."), hyperactivity/inattention (average $\alpha=.73$, range .71-.74; e.g., "Restless, overactive, cannot stay still for long."), and conduct problems (average $\alpha=.70$, range .68-.73; e.g., "Often fights with other children or bullies them.").

\section{Data Analyses}

Descriptive analyses were conducted to establish group leaders' fidelity to the intervention protocol and to assess parental attendance, quality of participation, and satisfaction with the program. One-way analyses of variance (ANOVAs) then examined whether groups differed from one another on $\mathrm{T} 1$ measures of child functioning and parenting practices in order to control for such differences if necessary. Finally, repeated-measures ANOVAs evaluated the extent to which parenting practices and child functioning changed following the program, as well as the magnitude of observed changes relying on partial $\eta^{2}$ (Richardson, 2011), and whether those changes were related to the number of program sessions parents attended.

\section{Results}

Fidelity

Relying on procedures described in Dumas et al. (2001), we recorded all sessions to monitor groupleader fidelity. Two trained coders listened independently to a random sample of $10 \%$ of the tapes and used checklists developed for the purpose to code each leader's fidelity to program process and content. Coders then compared their results and resolved differences in a consensual manner, listening again to critical tape segments as necessary. Results were used in supervision for ongoing feedback and provided overall estimates of adherence to protocol. On a 4-point scale, group leaders attained average process scores of 3.56 for their group management skills (range 3.45-3.64) and of 3.87 for their effective communication skills (range 3.68-4.00), and covered an average of $75 \%$ of all content items (range 57-93\%). This average does not mean that group leaders failed to cover a quarter of the program's content. Rather, in $71 \%$ of cases these deviations from protocol stemmed from the fact that group leaders failed to respect the time allotted to each program activity (i.e., gave some activities more time than the protocol allows, thus having to cover other activities more quickly than they should have).

Attendance, Quality of Participation, and Program Satisfaction

We used descriptive analyses to test hypothesis 1 , which concerned our expectation that parents would attend the program regularly, take part in sessions actively, and express high levels of satisfaction with the program. The 10 Entre-Parents groups we offered had between 6 and 17 parents each. Attendance was high, as the 130 parents came to an average of 6.6 of the 8 sessions $(S D=1.7$, range 1-8). Figure 1 shows that attendance was highly skewed: about $5 \%(n=7)$ of parents attended one or two sessions only, whereas $89 \%(n=106)$ of parents attended five or more sessions and $66 \%(n=86)$ attended seven or eight sessions.

Parents' quality of participation in sessions was also high and was not correlated with the number of sessions they attended $(r=-.09, n s)$. On a 5-point scale, all parents received average ratings of 3 or higher, and $21.4 \%$ received ratings of 4 or higher $(M=3.78, S D=.28)$. In keeping with these ratings, parents expressed high levels of satisfaction with the program. Table 2 showed that, on a 5-point scale, their ratings ranged between 4 and 5 on each of the three dimensions of program satisfaction we measured.

\section{Program Outcomes}

To take into account the fact that parents were nested within groups, we conducted one-way ANOVAs to

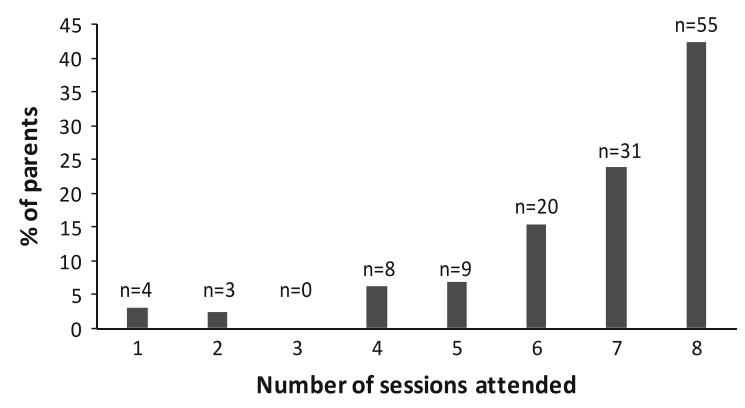

Fig. 1 Percentage of parents attending total number of sessions $(N=130)$ 
assess whether groups differed on any of the outcome variables at T1. Table 3 shows that the groups were comparable on all but two of those variables (i.e., PPI Harsh/inconsistent discipline and ECBI problem scale), for which we controlled in subsequent analyses.

Repeated-measures ANOVAs were conducted to test hypotheses 2 and 3 that program attendance would yield significant benefits in parental/family functioning and child adjustment over time (within-subjects variable), and that those benefits would reflect the number of sessions parents attended (between-subjects variable). These analyses compared overall mean changes across $\mathrm{T} 1, \mathrm{~T} 2$, and $\mathrm{T} 3$ in these variables, as well as mean changes as a function of attendance.

As hypothesis 2 predicted, Table 4 shows that there were significant main effects for time for four of the seven measures of parental/family functioning. Specifically, program benefits were evident in parenting practices, as parents reported using less harsh/

Table 3 Preliminary analyses to control for group differences on outcome variables at $\mathrm{T} 1$

\begin{tabular}{|c|c|}
\hline & $F(2,95)$ \\
\hline \multicolumn{2}{|l|}{ Parental/family functioning } \\
\hline \multicolumn{2}{|l|}{ Parenting Practices Interview (PPI) } \\
\hline Positive/appropriate discipline & 0.78 \\
\hline Harsh/inconsistent discipline & $2.13 *$ \\
\hline \multicolumn{2}{|l|}{ Parenting Stress Index (PSI-36) } \\
\hline Parental distress & 1.09 \\
\hline Parent-child dysfunctional interaction & 1.91 \\
\hline Difficult child & 1.27 \\
\hline \multicolumn{2}{|c|}{ Family Adaptation and Cohesion Scales (FACES-III) } \\
\hline Adaptability & 1.44 \\
\hline Cohesion & 1.69 \\
\hline \multicolumn{2}{|l|}{ Child adjustment } \\
\hline \multicolumn{2}{|c|}{ Social Competence and Behavior Evaluation (SCBE-30) } \\
\hline Social competence & 1.15 \\
\hline Anger-aggression & 1.21 \\
\hline Anxiety-withdrawal & 0.71 \\
\hline \multicolumn{2}{|l|}{ Eyberg Child Behavior Inventory (ECBI) } \\
\hline Intensity scale & 0.71 \\
\hline Problem scale & $2.19 *$ \\
\hline \multicolumn{2}{|c|}{ Strengths and Difficulties Questionnaire (SDQ) } \\
\hline Prosocial behavior & 0.68 \\
\hline Hyperactivity/inattention & 1.84 \\
\hline Conduct problems & 1.35 \\
\hline
\end{tabular}

inconsistent and more positive/appropriate discipline over time. However, there was a reduction in positive/ appropriate discipline over the follow-up period, with levels at T3 corresponding statistically to what they were at T1. Program participation was also associated with decreases in parental perceptions that their child was difficult and in-family adaptability. Although this latter decrease may appear undesirable, it actually indicates better structured yet flexible role relationships in the family, as high scores on this measure reflect chaotic family functioning in which role relationships are unclear (Olson, 2000). The effect size is given by the partial $\eta^{2}$ in order to determine the strength of the association.

Table 4 shows that program benefits were also evident on seven of the eight measures of child adjustment. Over time, children's social competence increased as their problematic behavior decreased. This decrease was observed in all problem areas we measured (i.e., SCBE-30 anger-aggression and anxiety-withdrawal, and SDQ hyperactivity/inattention and conduct problems) and when problematic behavior was considered both in terms of its intensity or of the number of problems parents reported (i.e., ECBI intensity and problem scales). Parents reported these benefits at the end of the program, with the exception of the decrease in hyperactivity/inattention, which only became evident at follow up.

As predicted by hypothesis 3 , there were also significant time by attendance interactions for three measures of parental/family functioning. Parents who attended more sessions reported significant decreases in parental distress $[F(2,95)=5.28, p<.01]$, parentchild dysfunctional interaction $[F(2,95)=3.38$, $p<.05]$, and chaotic family functioning $[F(2$, $95)=5.09, p<.01]$.

\section{Discussion}

This initial evaluation shows that attendance, quality of participation, and satisfaction with Entre-Parents were high, and that parents and their children benefitted from the program. Almost 9 out of 10 parents attended five or more of the eight sessions and twothirds of parents attended seven or all eight sessions. At some of our centers, we had to close registrations before the program started, as the number of group participants exceeded what our experience shows is a 
Table 4 Outcome analyses: changes in group means (standard deviations in parentheses) across time in parental/family functioning and child adjustment

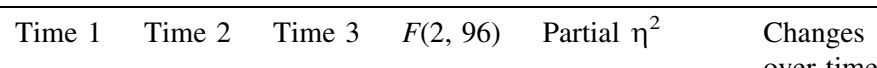
over time

Parental/family functioning

Parenting Practices Interview (PPI)

\begin{tabular}{|c|c|c|c|c|c|c|}
\hline \multirow[t]{2}{*}{ Positive/appropriate discipline $(1=\text { low, } 5=\text { high })^{\text {b }}$} & 4.06 & 4.25 & 4.14 & $8.64 * *$ & 0.15 , large $^{\mathrm{a}}$ & $\mathrm{T} 1<\mathrm{T} 2$ \\
\hline & $(.53)$ & $(.47)$ & $(.51)$ & & & $\mathrm{T} 2>\mathrm{T} 3$ \\
\hline \multirow[t]{2}{*}{ Harsh/inconsistent discipline $\left(1=\right.$ low, $5=$ high $^{\mathrm{b}}$} & 2.39 & 2.23 & 2.24 & $8.91 * *$ & 0.14 , large & $\mathrm{T} 1>\mathrm{T} 2$ \\
\hline & $(.50)$ & $(.43)$ & $(.52)$ & & & $\mathrm{T} 1>\mathrm{T} 3$ \\
\hline
\end{tabular}

Parental distress $(1=\text { low, } 5=\text { high })^{\text {b }}$

$\begin{array}{lllll}2.34 & 2.28 & 2.25 & 2.04 & 0.03\end{array}$

Parent-child dysfunctional interaction

$\left(1=\right.$ low, $5=$ high $^{\mathrm{b}}$

(.70)

(.71)

(.71)

$1.75 \quad 1.70$

1.69

(.43)

(.45)

(.52)

Difficult child $(1=\text { low, } 5=\text { high })^{\mathrm{b}}$

2.22

(.68)

(.74)

$1.31 \quad 0.04$

Family Adaptation and Cohesion Scale (FACES-III)

$$
\begin{aligned}
& \text { Adaptability }(1=\text { lax, } 5=\text { rigid })^{\mathrm{b}} \\
& \text { Cohesion }(1=\text { low, } 5=\text { high })^{\mathrm{b}}
\end{aligned}
$$

$2.89 \quad 2.76$

2.72

(.64)

Child adjustment

$\begin{array}{lll}6.29 * & 0.10, \text { moderate } & \begin{array}{l}\mathrm{T} 1>\mathrm{T} 2 \\ \mathrm{~T} 1>\mathrm{T} 3\end{array} \\ 0.60 & 0.01 & \end{array}$

\begin{tabular}{|c|c|c|c|c|c|c|}
\hline \multirow[t]{2}{*}{ Social competence $(1=\text { low, } 6=\text { high })^{b}$} & 4.17 & 4.34 & 4.39 & $8.90 * *$ & 0.14 , large & $\mathrm{T} 1<\mathrm{T} 2$ \\
\hline & $(.60)$ & $(.55)$ & $(.65)$ & & & $\mathrm{T} 1<\mathrm{T} 3$ \\
\hline \multirow[t]{2}{*}{ Anger-aggression $(1=\text { low, } 6=\text { high })^{\mathrm{b}}$} & 3.06 & 2.71 & 2.71 & $22.21 * *$ & 0.29 , large & $\mathrm{T} 1>\mathrm{T} 2$ \\
\hline & $(.73)$ & (.64) & $(.74)$ & & & $\mathrm{T} 1>\mathrm{T} 3$ \\
\hline \multirow[t]{2}{*}{ Anxiety-withdrawal $(1=\text { low, } 6=\text { high })^{\mathrm{b}}$} & 2.18 & 2.07 & 1.98 & $7.93 * *$ & 0.15 , large & $\mathrm{T} 1>\mathrm{T} 2$ \\
\hline & $(.63)$ & $(.60)$ & $(.52)$ & & & $\mathrm{T} 1>\mathrm{T} 3$ \\
\hline \multicolumn{7}{|l|}{ Eyberg Child Behavior Inventory (ECBI) } \\
\hline \multirow[t]{2}{*}{ Intensity scale $(1=\text { low, } 7=\text { high })^{\mathrm{b}}$} & 3.11 & 2.94 & 2.95 & $5.30 *$ & 0.11 , moderate & $\mathrm{T} 1>\mathrm{T} 2$ \\
\hline & $(.73)$ & (.69) & $(.79)$ & & & \\
\hline \multirow[t]{2}{*}{ Problem scale $\left(1=\right.$ no problem, $2=$ problem $^{\mathrm{b}}$} & 1.30 & 1.24 & 1.25 & $7.47 *$ & 0.13 , moderate & $\mathrm{T} 1<\mathrm{T} 2$ \\
\hline & $(.20)$ & $(.19)$ & $(.21)$ & & & $\mathrm{T} 1<\mathrm{T} 3$ \\
\hline \multicolumn{7}{|l|}{ Strength and Difficulties Questionnaire (SDQ) } \\
\hline \multirow[t]{2}{*}{ Prosocial behavior $(1=\text { low, } 3=\text { high })^{\mathrm{b}}$} & 2.59 & 2.59 & 2.64 & 1.22 & 0.03 & \\
\hline & $(.39)$ & $(.37)$ & $(.35)$ & & & \\
\hline \multirow[t]{2}{*}{ Hyperactivity/inattention $(1=\text { low, } 3=\text { high })^{\mathrm{b}}$} & 1.69 & 1.69 & 1.60 & $4.21 *$ & 0.08 , moderate & $\mathrm{T} 1>\mathrm{T} 3$ \\
\hline & $(.51)$ & $(.48)$ & $(.46)$ & & & $\mathrm{T} 2>\mathrm{T} 3$ \\
\hline \multirow[t]{2}{*}{ Conduct problems $(1=\text { low, } 3=\text { high })^{\mathrm{b}}$} & 1.70 & 1.59 & 1.59 & $7.50 *$ & 0.12 , moderate & $\mathrm{T} 1>\mathrm{T} 2$ \\
\hline & $(.41)$ & $(.41)$ & $(.40)$ & & & $\mathrm{T} 1>\mathrm{T} 3$ \\
\hline
\end{tabular}

Social Competence and Behavior Evaluation (SCBE-30)

As the groups differed on two variables at T1 (PPI harsh/inconsistent discipline and ECBI problem scale), the analyses reported here controlled for those variables

$* p<.05 ; * * p<.001$

${ }^{\text {a }}$ See Cohen (1988, p. 283: 0.0099 constitutes a small effect, 0.0588 a moderate effect, and 0.1379 a large effect)

b Possible responses on this scale 
satisfactory number to give all parents opportunities to participate in discussion and support each other. This high level of attendance compares favorably with parental attendance in the English and Spanish versions of PACE evaluated in the United States, where attendance was lower (Begle \& Dumas, 2011; Dumas et al., 2011), and contrasts with the common observation that "low levels of enrollment and attendance in parent training programs present major problems for researchers and clinicians" (Baker, Arnold, \& Meagher, 2011, p. 126). As we discuss below, this is likely to reflect important sociodemographic differences between the Swiss and the two American samples studied. We speculate that it may also reflect an important cultural consideration: Swiss parents are probably as reluctant as American parents to commit to an 8-week intervention. However, when they do, they do so with a strong sense of responsibility that they will honor by attending as regularly as they can. Irrespective of number of sessions attended, quality of participation was also high. Group leaders and assistants rated a majority of parents as interested in the topics covered in each session and actively involved in group discussions and activities. Not surprisingly, parents expressed high levels of program satisfaction: they rated group leaders and assistants very positively, found the session topics relevant and beneficial, and reported that they would recommend the program to others.

Considered together, these results support our first hypothesis that parents would attend Entre-Parents regularly, participate actively in sessions, and express high levels of satisfaction with the program. Support for this hypothesis was also found elsewhere. Many parents thanked staff often for the program and, when unable to attend a session, called in advance to apologize, let staff know that they would come back the following week, and request the handouts that group leaders distribute to summarize key points covered in sessions. In supervision, staff also reported that some parents spontaneously decided to get together with their children between sessions and, consequently, that they observed new friendships develop in the course of the program. Speculating on the major factor that might explain the success of our recruitment efforts and the resulting high level of program attendance, we believe that many parents decided to enroll in the program, not only because they heard about it through posters and flyers, but also because they were personally approached by program and preschool/daycare staff who answered their questions and encouraged them to enroll. In addition, prior to any recruitment, we organized a meeting at each center with its director and staff to describe the program in detail, answer questions, and make practical arrangements to recruit families and host the sessions, thus insuring as much as possible that center staff were strongly committed to the program and willing to recommended it to parents.

Quality of participation and program satisfaction in Entre-Parents were comparable to what we found in the English and Spanish versions of the program (Begle \& Dumas, 2011 Dumas et al., 2011). However, attendance was higher than in these two versions, especially the Spanish one. This is likely to reflect important sociodemographic differences between the Swiss and the two American samples studied. The Swiss sample was better educated overall and had higher family incomes than its American counterparts. Consequently, although it included an important proportion of recently immigrated families (like the Spanish sample), it probably benefitted from greater social support and material resources that made it easier for parents to attend sessions regularly. We note also that the Swiss sample of children included a higher proportion of males than females (unlike the American samples). This suggests that, in the preschool years, Swiss parents may be especially likely to seek guidance and support when they are concerned about the social and emotional development of their young boys. If this is correct, this concern may reflect the well-established finding that boys tend to present more challenging, especially disruptive, behaviors than girls in the early years (LaFreniere \& Dumas, 1996).

We also found strong support for our second hypothesis, as parents reported significant program benefits for themselves and their children. We obtained moderate to large effects over time on four of seven measures of parent/family functioning and on seven of eight measures of child adjustment. Results indicate that the program contributed to more effective parenting practices, a reduction in parenting stress, and an increase in family adaptability over time. (Family cohesion did not change significantly over time, possibly because it was already high at Time 1.) As one would have expected, parents also reported gains in their children's social competence and 
reductions in their children's disruptive and anxious behaviors, suggesting that, in the preschool years, one can effectively contribute to children's behavioral and emotional adjustment by guiding and supporting parents in their parenting task. (The children's prosocial behavior, as assessed by the $S D Q$, did not improve significantly over time, probably again because of a ceiling effect.) Finally, as we found in the English and Spanish versions of the program, our results indicate that some of the program benefits were stronger for parents who attended more sessions. Specifically, high attenders reported significant reductions on two of our three measures of parenting stress, as well as a reduction in chaotic family functioning. This finding suggests that intervention "dosage" may be an important component of program success, as most parents attended a majority of sessions in this study.

\section{Limitations}

The results just summarized are encouraging but must be interpreted with caution. First, the quasi-experimental study design makes it impossible to attribute the positive changes we observed to Entre-Parents. The fact that parents reported positive changes on a majority of measures and that some of these changes were greater for parents who attended more sessions suggest that the program may be efficacious, but alternative explanations, such as social desirability or child maturation, cannot be ruled out with this design. Second, we took care to offer the program in centers located across the French-speaking part of Switzerland but, because our sample was self-selected, we do not know the extent to which it is representative, especially of families in need of parenting guidance and support. Nor do we know what percentage of parents eligible to enroll in the program at each center actually did. (Studies suggest that the percentage may be relatively low, as many eligible parents may not see a need to participate in a preventive parenting program; Dumas, Nissley-Tsiopinis, \& Moreland, 2007; Heinrichs, 2006). Consequently, we caution against any generalization of our findings to other French-speaking families in or beyond Switzerland. Third, although we were very encouraged by the interest that many fathers showed for the program and by their active participation, the majority of our sample consisted of mothers. This gender imbalance, which is widespread in the literature and commonly ascribed to perceived barriers such as gender roles and work schedules, implies that our results are likely to apply to mothers of preschoolers more than fathers. Fourth, our measurement plan relied mostly on parental reports of parental/family functioning and child adjustment. This is common in the evaluation of new programs, as control or comparison groups are costly and rarely feasible because of practical difficulties concerning recruiting comparison families and assessing them at three points in time without offering them any intervention (Begle \& Dumas, 2011; Dumas et al., 2011). In that regard, we did consider (a) randomly assigning centers to our intervention or to a control, measurement-only condition, and (b) comparing our intervention to another similar program. However, preschool/daycare center directors strongly objected to a measurement-only condition and we could not find a suitable comparison program available in French. Future evaluations of Entre-Parents will need to include data from other sources of information to improve the robustness of their findings and to rely on randomized controlled designs to compare the program to another active intervention (e.g., Malti, Ribeaud, \& Eisner, 2011).

Another study limitation stems from the fact that the internal consistency of some of our measures fell below .70, which may have impacted our findings. This is likely to reflect, at least in part, the fact that we had to translate measures from English to French in order to evaluate the program. Future research will need to validate these measures further to determine how their reliability may be improved once it is clear that they are applicable to French-speaking respondents. Finally, as is true of our earlier evaluations of the English and Spanish versions of the program, our results are only relevant to similar universal prevention settings. They may not generalize to settings in which parents of children with behavioral and/or emotional problems are targets of intervention.

\section{Implications for Prevention Science and Services}

Entre-Parents was developed to offer a research-based parenting program to French-speaking parents of preschoolers. Like its English and Spanish counterparts, it was designed from the outset to be costeffective, as it is relatively short, delivered in a group format that maximizes opportunities for parental participation and support, and implemented by 
paraprofessionals (who must undergo a relatively short training and be regularly supervised). These features should make it attractive, both to researchers willing to independently evaluate its acceptability, efficacy, and cost, and to interventionists looking for a program that is informed by sound research and is manualized so that it can be implemented with fidelity. If these features are desirable, however, considerable research is needed to bring the program to scale, evaluate its effectiveness, and assess its cost by comparing it to other interventions offered to parents of young children. These substantial challenges are manageable, and must be overcome if our field is to make sound parenting programs much more widely available than they are today in order to guide and support parents in their task of bringing up socially and emotionally competent children.

Acknowledgments We wish to thank all the parents and professionals who participated in this program evaluation for their invaluable help. The study was supported by research Grants from the Jacobs Foundation (Zurich), the Swiss Office fédéral des migrations, ODM (through the Service de la cohésion multiculturelle, Canton of Neuchâtel), and Webster University (Geneva).

\section{References}

Abidin, R. R. (1997). Parenting Stress Index: A measure of the parent-child system. In C. P. Zalaquett \& R. J. Wood (Eds.), Evaluating stress: A book of resources (pp. 277-291). Lanham, MD: Scarecrow Press.

Baker, C. N., Arnold, D. H., \& Meagher, S. (2011). Enrollment and attendance in a parent training prevention program for conduct problems. Prevention Science, 12, 126-138.

Begle, A. M., \& Dumas, J. E. (2011). Child and parental outcomes following involvement in a preventive intervention: Efficacy of the PACE program. Journal of Primary Prevention, 32, 67-81.

Boggs, S., Eyberg, S., \& Reynolds, L. (1990). Concurrent validity of the ECBI. Journal of Clinical Child Psychology, $19,75-78$.

Cohen, J. (1988). Statistical power analysis for the behavioral sciences (2nd ed.). Hillsdale, NJ: Lawrence Erlbaum Associates.

Desmet, H., \& Pourtois, J. P. (Eds.). (2005). Culture et bientraitance. Bruxelles: De Boeck.

Dumas, J. E., Arriaga, X., Begle, A. M., \& Longoria, Z. (2010). "When will your program be available in Spanish?" Adapting an early parenting intervention for Latino families. Cognitive and Behavioral Practice, 17, 176-187.

Dumas, J. E., Arriaga, X., Begle, A. M., \& Longoria, Z. (2011). Child and parental outcomes of a group parenting intervention for Latino families: A pilot study of the CANNE program. Cultural Diversity and Ethnic Minority Psychology, 17, 107-115.

Dumas, J. E., \& Lucia, S. (2012). Promoting coping-competence in young children: Adaptation and translation of the PACE parenting program into French. Swiss Journal of Psychology, 71(2), 67-72.

Dumas, J. E., Lynch, A. M., Laughlin, J. E., Smith, E. P., \& Prinz, R. J. (2001). Promoting intervention fidelity: Conceptual issues, methods, and preliminary results from the EARLY ALLIANCE prevention trial. American Journal of Preventive Medicine, 20, 38-47.

Dumas, J. E., Moreland, A. D., Gitter, A., Pearl, A., \& Nordstrom, A. (2008). Engaging parents in preventive parenting groups: Do ethnic, socioeconomic, attitude, and value match between parents and group leaders matter? Health Education and Behavior, 35, 619-633.

Dumas, J. E., Nissley-Tsiopinis, J., \& Moreland, A. D. (2007). From intent to enrollment, attendance, and participation in preventive parenting groups. Journal of Child and Family Studies, 16, 1-26.

Dumas, J. E., Prinz, R. J., Smith, E. P., \& Laughlin, J. (1999). The EARLY ALLIANCE prevention trial: An integrated set of interventions to promote competence and reduce risk for conduct disorder, substance abuse, and school failure. Clinical Child and Family Psychology Review, 2, 37-53.

Geeraert, L., Van den Noortgate, W., Grietens, H., \& Onghena, P. (2004). The effects of early prevention programs for families with young children at risk for physical child abuse and neglect: A meta-analysis. Child Maltreatment, 9, 277-291.

Goodman, R. (1997). The Strengths and Difficulties Questionnaire: A research note. Journal of Child Psychology and Psychiatry, 38, 581-586.

Heinrichs, N. (2006). The effects of two different incentives on recruitment rates of families into a prevention program. Journal of Primary Prevention, 27, 345-365.

Kazdin, A. E. (2005). Parent management training. New York: Oxford University Press.

LaFreniere, P. J., \& Dumas, J. E. (1996). Social competence and behavior evaluation in children ages 3 to 6 years: The short form (SCBE-30). Psychological Assessment, 8, 369-377.

Malti, T., Ribeaud, D., \& Eisner, M. P. (2011). The effectiveness of two universal preventive interventions in reducing children's externalizing behavior: A cluster randomized controlled trial. Journal of Clinical Child and Adolescent Psychology, 40, 677-692.

Olson, D. H. (2000). Circumplex model of marital and family systems. Journal of Family Therapy, 22, 144-167.

Olson, D. H., Portner, J., \& Lavee, Y. (1985). FACES-III. University of Minnesota, Family Social Science. St. Paul, $\mathrm{MN}$.

Piquero, A. R., Farrington, D. P., Welsh, B. C., Tremblay, R., \& Jennings, W. G. (2009). Effects of early family/parent training programs on antisocial behavior and delinquency. Journal of Experimental Criminology, 5, 83-120.

Prinz, R. J. (2012). Effective parenting to prevent adverse outcomes and promote child well-being at a population level. In D. G. Mick, S. Pettigrew, C. Pechmann, \& J. L. Ozanne (Eds.), Transformative consumer research for personal and collective well-being (pp. 585-598). New York: Routledge/Taylor \& Francis. 
Richardson, J. T. E. (2011). Eta squared and partial eta squared as measures of effect size in educational research. Educational Research Review, 6, 135-147.

Rubin, K. H., \& Chung, O. B. (Eds.). (2006). Parenting beliefs, behaviors, and parent-child relations: A cross-cultural perspective. New York: Psychology Press.

Sanders, M. R. (1999). Triple P-Positive Parenting Program: Towards an empirically validated multilevel parenting and family support strategy for the prevention of behavior and emotional problems in children. Clinical Child and Family Psychology Review, 2, 71-89.

Sapin, M., Spini, D., \& Widmer, E. (2007). Les parcours de vie. De l'adolescence au grand âge. Lausanne: Savoir Suisse.

Serketich, W. J., \& Dumas, J. E. (1996). The effectiveness of behavioral parent training to modify antisocial behavior in children: A meta-analysis. Behavior Therapy, 27, 171-186.
Webster-Stratton, C. (1998). Preventing conduct problems in Head Start children: Strengthening parenting competencies. Journal of Consulting and Clinical Psychology, 66, 715-730.

Widmer, E. D., Kellerhals, J., Levy, E., Ernst, M., \& Hammer, R. (2003). Couples contemporains-Cohésion, régulation et conflits. Bern: Seismo Verlag.

Zisser, A., \& Eyberg, S. M. (2010). Treating oppositional behavior in children using parent-child interaction therapy. In A. E. Kazdin \& J. R. Weisz (Eds.), Evidence-based psychotherapies for children and adolescents (2nd ed., pp. 179-193). New York: Guilford. 